\title{
Challenges and Solutions in translating sepsis guidelines into practice in resource-limited settings
}

\author{
Suchitra Ranjit ${ }^{1}$, Niranjan Kissoon ${ }^{2}$ \\ ${ }^{1}$ Senior Consultant and Head, Pediatric ICU, Apollo Children's Hospital, Chennai, India; ${ }^{2}$ Children's and Women's Global Health, UBC \& BC \\ Children's Hospital Professor in Critical Care - Global Child Health, Department of Pediatrics and Emergency Medicine, UBC, Child and Family \\ Research Institute, Vice President Global Sepsis Alliance, Vancouver, Canada \\ Contributions: (I) Conception and design: Both authors; (II) Administrative support: None; (III) Provision of study materials or patients: None; (IV) \\ Collection and assembly of data: None; (V) Data analysis and interpretation: None; (VI) Manuscript writing: Both authors; (VII) Final approval of \\ manuscript: Both authors. \\ Correspondence to: Niranjan Kissoon, MB BS, FRCP(C), FAAP, MCCM, FACPE. Executive Medical Director, Children's and Women's Global \\ Health, UBC \& BC Children's Hospital Professor in Critical Care - Global Child Health, Department of Pediatrics and Emergency Medicine, \\ UBC, Clinical Investigator, Child and Family Research Institute, Vice President Global Sepsis Alliance, B245-4480 Oak Street, Vancouver, BC V6H \\ 3V4, Canada. Email: nkissoon@cw.bc.ca.
}

\begin{abstract}
Sepsis and septic shock are major contributors to the global burden of disease, with a large proportion of patients and deaths with sepsis estimated to occur in low- and middle-income countries (LMICs). There are numerous barriers to reducing the large global burden of sepsis including challenges in quantifying attributable morbidity and mortality, poverty, inadequate awareness, health inequity, underresourced public health, and low-resilient acute health care delivery systems. Context-specific approaches to this significant problem are necessary on account of important differences in populations at-risk, the nature of infecting pathogens, and the healthcare capacity to manage sepsis in LMIC. We review these challenges and propose an outline of some solutions to tackle them which include strengthening the healthcare systems, accurate and early identification of sepsis the need for inclusive research and context-specific treatment guidelines, and advocacy. Specifically, strengthening pediatric intensive care units (PICU) services can effectively treat the life-threatening complications of common diseases, such as diarrhoea, respiratory infections, severe malaria, and dengue, thereby improving the quality of pediatric care overall without the need for expensive interventions. A thoughtful approach to developing paediatric intensive care services in LMICs begins with basic fundamentals: training healthcare providers in knowledge and skills, selecting effective equipment that is resource-appropriate, and having an enabling leadership to provide locationappropriate care. These basics, if built in sustainable manner, have the potential to permit an efficient pediatric critical care service to be established that can significantly improve sepsis and other critical care outcomes.
\end{abstract}

Keywords: Low- and middle-income countries (LMIC); sepsis; shock; antibiotics; fluids

Submitted Sep 24, 2020. Accepted for publication Feb 05, 2021.

doi: $10.21037 /$ tp-20-310

View this article at: http://dx.doi.org/10.21037/tp-20-310

\section{Introduction}

The burden of infectious diseases disproportionately affects populations in low- and middle-income countries (LMIC) with a corresponding prevalence of sepsis. In these settings delivery of health care is generally suboptimal resulting in high death rates $(1,2)$.

Regardless of the underlying etiology, the 'sepsis syndrome', defined by the presence of severe infection with organ-dysfunction, is a common pathway contributing to this mortality. In patients with sepsis, multi-organ dysfunction 
leading to persistent end organ damage and refractory hypotension is associated with increasing deaths $(3,4)$.

The global burden of sepsis is undoubtedly massive (accounting for $20 \%$ of all deaths) with $90 \%$ of the annual 48.9 million cases and 11 million deaths in 2017 occurring in LMIC, where the barriers to improving outcomes are numerous (1).

Available data on sepsis management of patients across all age-groups in resource-limited settings (RLS) suggest that the high death rates is associated with sub-optimal management including delayed and inappropriate empiric antimicrobial therapy as well as ineffective initial resuscitation. Thus, in order to reduce sepsis mortality, a focus on improved management in these settings is urgently needed (2).

Worldwide, although $>90$ percent of the pediatric sepsis burden lies in LMIC and sepsis is a leading cause of maternal and childhood mortality $(1,5)$; sepsis remains a neglected killer without adequate recognition of its overarching contribution to childhood mortality $(6,7)$.

Moreover, the real burden of sepsis is very difficult to ascertain when clinicians rarely document it in their daily practice. For instance, although malaria, pneumonia and diarrheal diseases share sepsis as their final pathway to death, there has been very little emphasis on programs that specifically address sepsis. Patients are often classified based on the primary infective source, such as urinary tract infection or pneumonia, but sepsis is rarely included or considered in the diagnosis, and the use of nonspecific terms such as "septicemia" remains unfortunately pervasive (8).

The World Health Organization (WHO) has ,recognized sepsis as a global health priority which accounts for many millions of preventable deaths every year, and in 2017 adopted a resolution targeting the prevention, diagnosis, and treatment of sepsis, especially in LMIC (9).

This article aims to briefly outline the obstacles to optimal sepsis care in resource-limited settings (RLS), identify the knowledge gaps and inherent limitations in international definitions and treatment guidelines, and suggest broad avenues for improvement while acknowledging the fallacy of overgeneralizing across massively diverse geographies.

We provide suggestions how critical care resources can be made more accessible to a larger LMIC population, describe a suggested pragmatic approach to manage sepsis, and highlight key issues and research agenda that must be addressed to further refine best practice for the management of sepsis in these challenging settings.

We conclude with a brief outline of possible future application of creative, rapidly-evolving modern low-cost technological innovations that hold potential to advance LMIC closer to equity in the global healthcare arena.

\section{Barriers and deficiencies in LMIC that contribute to preventable sepsis deaths ( Table 1) $(8,10,11$ )}

The enormous burden of childhood mortality in LMIC is tragic considering that many deaths could be prevented by timely, simple resuscitative measures despite austere environments that lack critical care services $(6,7,12-14)$.

The challenges and difficulties in LMICs includes severely limited government spending on public health, inadequate coverage with vaccines, poor sanitation and water, inadequate primary education which results in higher prevalence of diseases and poorer outcomes including that for sepsis $(8,15)$.

Even when care for sepsis is sought, further obstacles to appropriate care are numerous, and include poorlyequipped transport to an appropriate medical facility, rudimentary or no triage and non-availability of an organized screening for sepsis or institution of critical care support. Major contributors are also lack of trained medical and nursing personnel and equipment for emergency care including the administration of timely antibiotics and resuscitation, which poses a greater challenge in resourcelimited settings in LMICs than wealthier regions (16).

Challenges in provision of quality treatment might also include counterfeit antimicrobials, limited surgical and obstetrical interventions, and intensive care that might or might not have life support interventions or protocols for managing patients with physiologic derangements $(16,17)$.

\section{Limited understanding of epidemiology and pathogenesis of sepsis in developing countries}

Limited understanding of the epidemiology and pathogenesis in LMICs stems from the fact that the majority of published experience are from large, often academic urban centres, rather than rural or district level healthcare facilities where most patients present. Furthermore, there is a major lack of meaningful data about the risk factors for developing sepsis and how they should be managed especially from the lowest income countries where sepsis is most common and associated with the poorest outcomes $(6,18)$. It is difficult also to extrapolate data and generalize information reported from a few resourcelimited areas as being representative to all LMIC since the 
Table 1 Pediatric sepsis-summary of challenges and proposed solutions in resource limited regions $(8,10,11)$

\begin{tabular}{|c|c|c|}
\hline (I) Overarching challenges & (II) Cause/consequence & (III) Possible solutions \\
\hline $\begin{array}{l}\text { Inadequate acute and urgent } \\
\text { healthcare delivery systems }\end{array}$ & Poverty, political corruption, health inequity & $\begin{array}{l}\text { Sustained efforts to fortify public health services and also } \\
\text { acute health care delivery }\end{array}$ \\
\hline Limited human resources & $\begin{array}{l}\text { Inadequate healthcare personnel, major } \\
\text { knowledge gaps in healthcare providers' } \\
\text { training that delay timely identification and } \\
\text { management of sepsis }\end{array}$ & $\begin{array}{l}\text { Adequately staffed and well-supplied referral centres } \\
\text { accessible to all. } \\
\text { Ongoing training programmes }\end{array}$ \\
\hline $\begin{array}{l}\text { Rampant anti-microbial } \\
\text { resistance }\end{array}$ & $\begin{array}{l}\text { Increased ESBL rates leading to greater high- } \\
\text { end antibiotic use and further resistance }\end{array}$ & Focus on prevention of hospital-acquired infection \\
\hline $\begin{array}{l}\text { Inadequate quantification of } \\
\text { sepsis burden in LMIC }\end{array}$ & $\begin{array}{l}\text { Wide variations in case definitions developed } \\
\text { in HIC with uncertain utility in LMIC }\end{array}$ & $\begin{array}{l}\text { Locally relevant operationalization of sepsis definitions, } \\
\text { including greater emphasis on clinical criteria and simple } \\
\text { vital signs rather than laboratory criteria to define sepsis }\end{array}$ \\
\hline \multirow[t]{2}{*}{$\begin{array}{l}\text { Lack of relevance in } \\
\text { translating high-resource } \\
\text { sepsis treatment guidelines } \\
\text { in LMIC }\end{array}$} & $\begin{array}{l}\text { Different genetic features of immune response } \\
\text { Different comorbidities such as HIV, } \\
\text { malnutrition }\end{array}$ & \multirow[t]{2}{*}{$\begin{array}{l}\text { Local testing of international treatment guidelines using } \\
\text { available resources. }\end{array}$} \\
\hline & $\begin{array}{l}\text { Different invading pathogens: parasitic, viral, } \\
\text { mycobacteria }\end{array}$ & \\
\hline
\end{tabular}

LMIC, low- and middle-income countries; HIC, high income countries; ESBL, extended spectrum beta lactamase; HIV, human immunodeficiency virus.

host characteristics, sepsis etiology and pathogenesis are likely to vary a great deal across the enormous range of regions classified in the LMIC category (19).

\section{Varying spectrum of causative agents and pathophysiology}

The etiology of sepsis in LMICs is diverse and poses difficulties because of unique causative pathogens in these mostly tropical countries, limited epidemiological data because of inadequate capacity of microbiological services, leading to poor antibiotic stewardship and a higher degree of AMR.

There is a wide-range of pathogens in LMIC, and while gram-negative and gram-positive bacterial etilogies are fairly common, other bacteria that that are infrequently encountered in HIC but commonly result in the sepsis syndrome in Asia include Burkholderia pseudomalle, Rickettsiosis and salmonella infections which may be responsible for melioidodis, scrub typhus and enteric fever respectively $(16,20,21)$.

Further, acute non-bacterial diseases may be often be responsible for sepsis including protozoal diseases such as malaria, and viral diseases such as measles, dengue, or viral hemorrhagic fevers (17). Non-bacterial causes are understudied and under-represented, and the 
pathophysiology and treatment of bacterial sepsis may not be generalizable to these other causes of sepsis

While non-bacterial etiology may also result in a dysregulated host response and organ failure, other mechanisms of organ failure include potential direct damaging effects of certain pathogens or pathogen products, which can in particular play a role in tropical diseases. For instance, in severe falciparum malaria, both micro- and macrovascular dysfunction may be contributory factors and it has been well established that an infected and sequestered red blood cell biomass can obstruct the microcirculation and directly cause of vital organ failure $(22,23)$. Similarly, in severe dengue, the viral proteins may directly cause endothelial glycocalyx damage leading to plasma leakage and shock, and organ failure can also occur due to direct virus invasion (24).

\section{Widespread anti-microbial resistance}

Although the bacterial pathogens that cause sepsis in LMICs are similar to those reported in high-income countries (HIC), antimicrobial resistance (AMR) patterns can be very different. Increasing reports of the high prevalence of multidrugresistant bacteria, including extended spectrum betalactamase (ESBL)- producing bacteria, methicillin-resistant Staphylococcus aureus, resistant Mycobacterium tuberculosis, and Enterobacteriaceae producing carbapenamase are of concern and may contribute to the high mortality observed in LMICs particularly among infants $(8,17)$.

Levels of Gram-negative bacterial resistance in LMIC are alarmingly high, particularly in Asia, where hospitalacquired ESBL rates above 60 and $80 \%$ in India and China respectively have been reported (25). The highESBL rates leads to increased carbapenem use in these regions with selection pressures resulting in the appearance of carbapenem resistance (26). Five countries with the highest burden of under-five deaths (Nigeria, India, China, Pakistan, and the Democratic Republic of the Congo) also have the greatest rates of neonatal deaths from AMR (27). The costs and quality of the medication are important factors guiding antibiotic choice and, although generic products are cheaper; the quality may be sub-standard or even counterfeit (28).

\section{Challenges in the application of pediatric sepsis definition and recognition in LMICs}

Sepsis is a complex heterogenous syndrome, the variable manifestations of which are determined by multiple factors including genetic, background chronic illness, nutritional status, pathology and invading organism, time for initiating therapy among others $(3,29)$. Despite the huge burden that sepsis imposes on the health of children, there remains a dynamic conflict between various iterations of the consensus criteria of sepsis and the criteria that clinicians use at the bedside to diagnosis sepsis or septic shock $(12,30)$.

On account of the non-specific physiologic abnormalities, there is discordance between the sepsis definitions and physician-diagnosed sepsis, both in wealthier and lesserresourced countries (31). We all agree on the definition which is a philosophical and physiological construct of presumed or suspected infection and end organ dysfunction. What is uncertain is how it is operationalized at the bedside because of varying levels of resources to confirm organ dysfunction $(15,32)$.

The recognition children at risk for sepsis or septic shock, even before advanced organ dysfunction is established and timely initiation of therapy may improve morbidity and/or mortality and assumes greater importance in LMIC regions where advanced organ support is limited or unavailable. However, the primary healthcare workers in LMIC who cater to all-comers including infants, older children and adults may not be educated or trained to recognize pediatric sepsis in the early stages when the clinical signs are subtle.

Even experienced pediatric providers in high-income countries may have difficulties in pediatric sepsis diagnosis and recognition. A study in a tertiary care children's hospital in US reported prevalent sepsis knowledge deficits, provider discomfort, and diagnostic delays among all provider groups (33).

The 2005 pediatric sepsis definition is outdated, has low specificity and sensitivity and is now being updated.

\section{Challenges in therapy of sepsis in LMIC}

Similar to the challenges in defining sepsis in LMIC, extrapolation of international treatment recommendations in LMIC is fraught with challenges.

\section{Antimicrobial therapy: the need to cover a broad range of etiological agents vs. practicing antibiotic stewardship in regions with major antimicrobial resistance}

There is a high degree of variation in the microbiological etiology of sepsis between and within resource-limited settings and the empiric antibiotic regimen for a patient 
who presents with suspected sepsis in LMIC must cater to local epidemiology and resistance patterns, including bacterial and or non-bacterial etiologies such as TB and malaria, and viral diseases such as dengue or measles.

Further, the limited microbiological laboratory capacity in many LMICs leads to difficulty in practicing antibiotic stewardship by de-escalation of the empiric broad antibiotic regimen based on exact etiology, further compounding the problem of antimicrobial resistance.

\section{Timing of antibiotics}

The Peds SSC recommendation aims to optimize antibiotic stewardship by attempting a balance between timely antibiotic therapy to optimize outcomes while simultaneously attempting to minimize antibiotic use in non-bacterial conditions. Accordingly, the recommendation is to provide antimicrobial therapy within 1 hour of recognition in the presence of septic shock, while in children without shock but with sepsis-associated organdysfunction, the suggestion is to start antimicrobial within 3 hours of recognition. This period will permit appropriate and expeditious diagnostic investigation for patients with an uncertain diagnosis in order to discriminate those with actual sepsis and those with suspected infection, and this approach is appropriate even in LMIC (3).

\section{Early resuscitatio: HIC model may not be relevant}

The overarching premise that governs early sepsis resuscitation protocols was that a time-based sepsis pathway that promotes aggressive use fluid resuscitation and vasoactive agents would improve both the quality of resuscitation thereby sepsis outcomes, whereas patients who received (slower) usual care were at higher risk of underresuscitation, with consequent poorer survival rates. The protocolized rapid resuscitation was in stark contrast to the pervasive practice of slow correction of deranged vital parameters that underpins usual care practices in many LMICs (34). However, and in contrast to expectation, the 3 African fluid studies in adults and children demonstrate that caution may in fact be warranted, and that the quest to rapidly normalize deranged parameters for patients with severe sepsis in resource-limited settings may be counterproductive (35-37).

An improved focus on identifying the priority interventions for sepsis management in RLS is a major challenge. While there is evidence in LMIC for the benefit of timely and appropriate antimicrobials, the utility of other interventions commonly practiced in HIC is difficult (8). For example, what are the risks $v s$. benefits of fluid resuscitation in the absence of vigilant patient monitoring and timely respiratory support? Similarly, are invasive central venous lines beneficial for guiding fluid resuscitation in a RLS considering the greater risks of infection and mechanical complications? Finally, given global efforts to improve oxygen therapy practices, what is the optimal level of oxygen saturation in septic patients? Clearly some interventions such oxygen in hypoxemic respiratory failure are universally useful while others such as fluids may have conflict.

\section{Fluid resuscitation: changing paradigms}

Since its introduction during the cholera epidemics of the nineteenth century, intravenous fluid resuscitation has served as a mainstay of supportive sepsis care (38). Today, there is increasing concern that intravenous fluids may unexpectedly augment septic endothelial dysfunction, potentially negating the beneficial hemodynamic effects of fluid resuscitation $(38,39)$.

All aspects initial fluid resuscitation in septic shock are extensively debated, and there is controversy regarding the volumes, rates, and type of fluid to be administered, and even whether the initial fluid bolus is required at all in every patient with septic shock. Fluid boluses can cause undesirable effects, and while pulmonary edema and fluid overload are best known, adverse effects can occur due to its vasodilating properties (40) and effects on the endothelial glycocalyx promoting worse capillary leak and increased lung fluid even without fluid overload (39). Fluid can cause respiratory deterioration and cardiovascular collapse in the presence of abnormal cardiac function (which may be present in $40-60 \%$ of patients) (3), which may lead to death if rescue ventilation is not immediately available. Large volume resuscitation may be harmful in children with underlying chronic illness such as TB, malaria, malnutrition and anemia.

Studies in even well-resourced countries demonstrate that the desired response to the fluid bolus in terms of improved cardiac output may be transient, with reports of $2 / 3$ rds of children with septic shock who received FB demonstrating an increased $\mathrm{CI}>10 \%$ at 5 minutes, of whom only $14 \%$ remained fluid-responsive at 60 minutes, suggesting that in the remaining patients the administered fluid might have leaked out into the interstitial compartment, causing short- 
lived benefit and unintended harm (41).

\section{There may not be a single ideal rate and volume of fluids to be administered to all children presenting with septic shock in resource-limited regions}

It is clear that there is no single best volume or rate of fluid resuscitation that will suit all patients presenting with septic shock in any area of the world, least of all in LMIC. While many children with septic shock may have decreased intake and increased losses, absolute volume deficits may not be large. Plasma leak may also occur to some degree in sepsis, but not to the extent seen in established capillary leak conditions such as dengue shock. Even in patients with severe dengue shock with clear evidence of capillary leakinduced hemo-concentration, pleural and ascitic collections and narrow pulse volume, fluid replacement comprising 20-25 mL/kg over the initial 2 hours, with an ongoing decreasing regimen depending on response is sufficient in most children (42).

In septic shock also, fluids deficits may not be large in the absence of clear fluid-losing state. Hypovolemia is more relative (from re-distribution in vasodilatory shock) with a minor degree of absolute hypovolemia (43). We have demonstrated that a more physiologic approach to improve preload in vasodilatory septic shock includes an initial small volume followed by agents such as low-dose pressors which improve the venous return (rather than large volume fluid boluses) (44).

\section{Respiratory/ventilatory support for patients with septic shock: challenges in LMIC}

Many patients with septic shock require oxygen and respiratory support either from their primary illness such as pneumonia or during the period of rapid fluid resuscitation.

There are many barriers to delivering high-quality support to patients with acute respiratory failure in RLS, chief among which include inadequate equipment that are poorly maintained, and understaffed health personnel who are inadequately trained (17).

The practice of intubation and mechanical ventilation is limited in LMIC and carries high crude mortality, with reported ICU death rates of between $36-72 \%$ (45). The risk of ventilator-associated pneumonia (VAP) and other complications are also greater in LMICs than in HIC with greater costs, length of stay and mortality (45).

\section{Looking ahead: WHAT are the ways to improve outcomes of critically ill septic children in LMIC}

\section{Advocacy required at many levels}

The UN sustainable development goals (SDG) have set ambitious targets to reduce neonatal and under- 5 mortality to less than 12 and 25 per 1,000 live births respectively, and universal access to vaccines and affordable medications $(8,17,46)$.

Advocacy is also required at the global, national, and regional levels to raise awareness in order to prevent infections, to increase access to medications and life-support equipment, and to ultimately build strong and resilient healthcare systems that will be able to provide sustained healthcare services even when infectious outbreaks occur (47). These global efforts will bring nations closer to reaching the SDG of attaining lower neonatal and under-5 mortality rates $(48,49)$.

The Global Sepsis Alliance is raising awareness at the international level on strategies to prevent sepsis as well as monitor, manage, and reduce morbidity and mortality, while simultaneously advocating for inclusion of sepsis as a separate cause of death in the Global Burden of Disease framework.

\section{Preventative measures}

Advocacy at national and regional levels requires national health policy makers to focus on not just poverty alleviation, but also prevention of the risk factors for high infant and under-5 mortality such as low-birth-weight, undernutrition, indoor air pollution, incomplete immunization at one year, HIV, lack of exclusive breast-feeding for the first 6 months, over-crowding and lack of hygiene, the lack of clean water and sanitation, maternal illiteracy, and challenges in accessing healthcare due to longer travel times (46,50,51).

Effective communication with government health managers and policy makers on a consistent basis is crucial in order to improve funding and support of the critically ill child, emphasizing that may diagnoses that contribute to the high burden of disease in LMIC, such as sepsis, dengue, poisoning, and diarrhoea are acute fully curable critical illnesses, and can be managed through prompt, simple lowcost treatments despite limited pediatric intensive care resources compared with HIC $(52,53)$.

Considering these key differences, there is a compelling public-health argument for basic intensive care services even in LIC given that even a short period of critical care is a cost-effective and essential part of the treatment of the 
acute life-threatening conditions that affect the lives of millions of young people worldwide. In a Malawi hospital, emergency department triage and treatment for children costs just US\$1.75 per patient and has greatly reduced hospital mortality and the cost of providing oxygen just 1-6 US dollars per day (54).

\section{Continuous public education to seek better bealth care}

To reduce $\mathrm{U} 5 \mathrm{M}$, basic education regarding early signs and symptoms of common infectious diseases should be repeatedly provided to caregivers so that they are able to easily recognize these and seek care promptly. In LMICs, the ability of caregivers to recognise common diseases such as diarrhea, pneumonia or malaria and their danger signs, and to seek health care promptly is very low. These issues are even more prominent in rural regions, where delays due to the use of traditional care practices is also of concern. Other factors influencing appropriate healthcare seeking include socioeconomic status and cost of care; severity of illness, and in some regions even the gender of the child with girls less likely to receive appropriate care (55).

\section{Prompt antimicrobials and oxygen}

Timely antimicrobials have repeatedly shown to improve sepsis outcomes in both HIC and LMIC. An analysis in 101 countries has shown that with an estimated 590,000 cases of pneumonia in under- 5 children, universal access to antimicrobials can prevent 445,000 deaths (14). However, many LMICs have limited access to even basic life-saving medications, which adds to the burden of preventable sepsis-associated deaths. Strong and sustained advocacy to increase universal access to life-saving medications can greatly reduce the massive burden of sepsis (56).

Supplemental oxygen has been shown to decrease death rates in childhood pneumonia by up to $35 \%$ and is listed on the WHO's essential medications (57). However a survey conducted among 97 physicians from 19 countries across Asia, Africa, and South America reported that less than a third of respondents reported working with uninterrupted oxygen supplies in their healthcare facilities (58).

\section{Universal vaccinations}

Vaccinations remain amongst the most cost-effective interventions to reduce sepsis mortality and their access in LMICs should be greatly improved. Further, antibiotic use may be greatly reduced with widespread vaccinations, and a recent analysis of 75 countries showed that with adequate access to vaccines for children under-5 years of age, an estimated 11.4 million antibiotic days can be averted each year with improvement in death rates $(27,59)$. Gavi, the international alliance that actively advocates and promotes improved immunisation, has partnered with LMIC governments to vaccinate 580 million children, thereby averting greater than 8 million deaths over the previous 15 years (60) (http://www.gavi.org/progressreport/\#section2).

Unfortunately, a growing section of the society has become vaccine-hesitant because of the risk-benefit concerns that are often inappropriately raised related to political motives and social media (61). Because of its negative influence on vaccination confidence, the availability of misinformation on social media should be identified as a threat to the public's health.

In a vaccine-hesitant atmosphere, sustained health education that supporting the government campaigns against vaccine refusal are essential in changing attitudes (62). This requires a climate of respect and mutual trust between science and society to be promoted, where scientific knowledge is not only taught but also cultivated and sustained coupled with an ongoing emphatic understanding of citizens worries, needs of reassurance and health expectations (62).

\section{Critical care is expensive! Can an inexpensive model be developed?}

The annual healthcare expenditure per capita across LMIC is only around $5 \%$ that of $\mathrm{HIC}$, and provision of critical care is rarely considered to be a priority in LMIC. Furthermore, in LMICs, critical care costs are only seldom covered by the health insurance and high out-of-pocket expenses may be incurred with each ICU admission $(8,16)$, leading to restricted access to ICU care for the socioeconomically disadvantaged and uninsured. Therefore, an urgent need to develop an alternative inexpensive yet effective critical care model for LMIC is required.

It is becoming increasingly obvious that high quality critical care in LMIC is unlikely to be achieved by efforts to replicate the HIC model based on resource-intensive ICUs with technologically advanced equipment and vast numbers of highly-trained staff. Rather, efforts to develop of contextappropriate solutions that are devised and championed by local healthcare professionals based on locally relevant 
evidence may be lead to far more successful outcomes $(52,63)$.

The WHO has published a pocketbook for the management of common pediatric illnesses (64) which provides clinical guidelines for hospitalized children in regions with limited resources. These guidelines have led to improvements in staff training and have clearly demonstrated that significant gains can be achieved in caring for critically ill children in LMICs by appropriate triage and rapid supportive treatment using inexpensive therapeutic modalities, including fluids, oxygen, and antimicrobial therapy. However, rapid recognition, triage and the ability to provide rapid supportive interventions still remains mostly inadequate in many LMIC settings leading to unabated high mortality even in common treatable infections such as pneumonia and malaria, further $90 \%$ of deaths from childhood trauma also occur in LMICs (65).

Thoughtful approaches are urgently required to develop Emergency and Critical Care Services in LMIC (66) and can impact outcomes if combined with a focus on caregiver recognition of serious illness, prompt access to care, safe and expedited transport and efficient triage (67).

Even when hospitals have facilities to deliver emergency care, often a lack of co-ordination, prioritisation, timely identification and institution of basic life-saving treatments can result in millions of salvageable lives lost (67). Baker et al. have proposed a new focus on Essential Emergency and Critical Care (EECC)-care that critically ill patients should receive in all healthcare facilities including LMIC (66). EECC-care should be part of universal health coverage, is appropriate for all countries in the world, and is intended for patients irrespective of age, gender, underlying diagnosis, medical specialty, or location in the hospital.

Well-organized EECC-care is pragmatic and low-cost and does not need to be excessively reliant on sophisticated technology, and can be developed to have a primary focus on simple, effective actions that have large potential impact on the population.

Locally relevant and user-friendly treatment protocols should be developed and implemented with WHO's leadership and through local authorities. The forefront role of oxygen therapy should be emphasised, uninterrupted oxygen supplies and delivery systems ensured along with guidelines for appropriate use. Other essential care includes a patient in head-up position, functioning suction, and basic chest physiotherapy, and these elements of care can easily be implemented by health workers with basic training (67).

Setting up EECC-care in a LMIC setting requires careful planning, region-specific resources, and most crucially, investment in the permanent healthcare providers, both nursing and medical $(53,65)$, and starts with the fundamentals: continuous training healthcare professionals in skills and knowledge, selecting resource-appropriate equipment, and having an enabling leadership to provide a supportive environment for appropriate care. If these fundamentals building blocks can be built in a sustainable manner, an resource-appropriate critical care service can be establish that has the potential to significantly reduce childhood mortality thus enabling LMIC to reach toward the sustainable development goals (SDG) (68).

Good quality essential critical care can have a large positive impact on mortality even without formal ICUs. Moreover, timely provision of essential supportive care can prevent progression to multi-organ dysfunction, thereby reducing the burden on limited ICU capacity (67).

Critical care is a limited resource all over the world, and more so in LMIC, and allocating these resources for the sickest patients who are most likely to benefit must be emphasized. Patients who have milder illnesses as well as those who are moribund must not be admitted to scarce ICU beds and should be cared for in general hospital wards or palliative-care units, respectively.

Further, it is essential to promote staff wellness so that the few and "precious" trained personnel are not overworked, emotionally ravaged and compelled to leave, further worsening a limited resource. Witnessing a high ICU mortality rate even in treatable conditions leads to personal and professional frustrations that could risk fatalism and staff burnout, leading to inappropriate therapy of even potentially salvageable patients (69). Attention to improved hospital functioning coupled with investment in clinician education, emotional support and counseling may improve the retention of trained healthcare providers and educators (69).

\section{How can critical care practitioners practice locally relevant intensive Care?}

\section{Critical care practitioners must make pragmatic modifications so that the care is locally relevant}

Given the paucity of good quality evidence regarding various interventions from LMIC for sepsis, there is a natural tendency to 'force-fit' existing time-based treatment protocols in vastly different LMIC environments. However, there is growing realization that recommended international intensive care interventions for the management of patients 
with sepsis may be impractical, unaffordable, and possibly even harmful when implemented in middle- and lowincome countries (68), and local critical care leadership in various regions must tailor pragmatic diagnostic and therapeutic approaches catering to a wide range of settings. We have outlined a few suggestions below.

\section{Striking a balance in sepsis screening, recognition, and definition in LMIC}

Screening tools and severity scores must enable effective triage and early sepsis recognition in LMIC, these will facilitate earlier treatment that is more likely to be successful with lesser resource utilization. However, there may be difficulties in implementing screening modalities developed by HIC and these may be impractical in resource-poor settings where laboratory diagnostic capacity is limited and most healthcare workers have minimal training.

In settings with high mortality rates, particularly in LMIC, the challenges in sepsis screening stem from the need for a shift from specificity toward sensitivity, whilst de-emphasizing the requirement for laboratory testing and with a therapeutic response graded according to severity of illness to permit earlier recognition. Front-line clinicians need robust screening tools rather than just riskstratification tools. Further, a separate treatment pathway must be offered for patients who are unwell but not yet sick enough to satisfy standard scoring criteria (70).

Although the initial support of a patient with sepsis can proceed even without identification of the pathogen but includes the administration of broad-spectrum antibiotics along with source control where appropriate, laboratory information and expensive-technology is not relevant in the early stages (8). This strategy highlights the need for sensitive criteria allowing early recognition of and intervention for sepsis, with an emphasis on a cliniciandefined spectrum of disease that relies on easily available data.

\section{Screening tools based on available resources: Using simple vital signs and deranged physiology}

The mainstay of critical care is monitoring vital signs (temperature, pulse rate, blood pressure, respiratory rate, mental status, oxygen saturation) and these can be implemented in hospitals without advanced monitoring facilities, and once identified, treating deranged physiology emergently may be lifesaving (71).
Deranged physiology indicates that the patient is at risk of acute deterioration, and are also termed as "Danger Signs" to emphasize the need for immediate intervention. The Danger Signs must be included in a simple to follow checklist in order to standardize the triage and streamline the initiation of early life saving therapies. For example, a checklist in Africa specifies that a patient with even a single Danger Sign (e.g., 'reduced conscious level') is classified as an emergency, and also indicates the investigations or treatments that should be initiated (14).

The most effective screening tools in LMIC emphasize clinical criteria rather than laboratory investigations, and among children, a study from Africa reported that largely clinical criteria and simple vital signs can be useful for screening patients where the admission burden is very high, to indicate those children at greatest risk of poor outcome, in order to prioritize intravenous antimicrobials and prompt supportive therapies. Severe forms of sepsis, pneumonia, or malaria were the most common causes of death in Africa (72), yet the differentiation of the specific diagnosis or pathogen is not clear at time of admission since they had similar clinical characteristics at presentation, furthermore, most of the deaths occurred within the first hospital day (73).

The authors have proposed a bedside clinical risk score, the FEAST-PET [FEAST Pediatric Emergency Triage (PET) score] for severely ill children presenting to emergency care wards in resource-limited settings in Africa, based on data collected during the Fluid as Expansive Supportive Therapy (FEAST) trial (72), and this score has been externally validated. The FEAST-PET score aims to identify patients at highest risk of death within 48 hours of admission based solely on bedside clinical parameters that can be rapidly identified by busy healthcare workers at admission, and does not need a specific disease/ infection to be identified, and that adequately discriminates between children at different mortality risk.

Recent studies in LMIC suggest that syndromic identification represents a crucial method to triage sick children appropriately, and a practical triage tool for children is in the process of validation by Fung et al. that can rapidly and reliably be used by low-skilled frontline healthcare workers to identify children at risk of sepsis without the need for extensive memorization or training (74).

\section{Advantages of SIRS in LMIC}

Although the systemic inflammatory response syndrome (SIRS) criteria have a poor discriminant validity, in LMIC, 
SIRS could still have a role in identifying patients with infections who may benefit from antibiotics, fluids and screening for organ dysfunction. Many quality improvement (QI programmes) have used this approach and have reported a positive impact on mortality reduction (15), and in the presence of a documented infection, the presence of 2 or more SIRS criteria may help to identify patients who need additional screening for organ dysfunction (8).

\section{Adapting sepsis- associated organ failure definitions to the existing health care system}

With respect to sepsis induced pediatric acute respiratory distress syndrome (PARDS), the definition is made when a child with hypoxemic respiratory failure requires invasive or non-invasive ventilation and is based on oxygenation indices and acute onset chest X-ray changes (75). However, when facilities to perform blood gases or provide positive end-expiratory pressure (PEEP) are not present, it may be difficult to even identify PARDS which may consequently be under-diagnosed, and appropriate management delayed.

Alternate diagnostic modalities may permit ARDS recognition in resource-limited settings, and a study from Rwanda reported the Kigali modification of the Berlin definition of adult ARDS that aimed to overcome the local diagnostic challenges by modifying the ARDS definition to the existing healthcare facility. The Kigali modification permits ARDS definition even without PEEP values, and hypoxia was defined with $\mathrm{SpO}_{2} / \mathrm{FIO}_{2}$ (SF ratio) less than or equal to 315 along with the presence of bilateral opacities at chest X-Ray or bedside lung ultrasound $(76,77)$.

While a disease definition aims to facilitate case recognition and match treatment options to severity and has the same clinical importance world over. The Kigali modification of the ARDS definition emphasizes the pragmatic adjustments that can facilitate critical care medicine even in resource-limited regions.

\section{Pediatric septic shock resuscitation: fluid bolus decision depends on resources available}

The most recent pediatric SSC has a differentiated approach to initial fluid resuscitation depending on the resources available at the health-care facility where the patient presents. In resource-poor settings without adequate critical care services, the Pediatric SSC recommend a careful, personalized approach to fluid bolus administration and for those without evidence of severely impaired circulation without access to ICU resources, only maintenance fluids administration is recommended. For patients with evidence of hypotensive shock suggesting a severely impaired circulation, a carefully monitored fluid bolus of $10-20 \mathrm{~mL} / \mathrm{kg}$ over 30 minutes is suggested (3).

The WHO regimen is also similarly cautious and suggests that the initial bolus of $10-20 \mathrm{~mL} / \mathrm{kg}$ of isotonic crystalloid (balanced crystalloid preferred, if unavailable normal saline) may be infused over 30-60 minutes depending on the severity of shock/dehydration (78).

The effectiveness of oral rehydration therapy in severe diarrhoeal dehydration shows that the gastrointestinal tract is a potentially useful route of fluid resuscitation, even in extreme intravascular depletion, and merits evaluation in sepsis (12).

\section{A monitored and individualized septic shock resuscitation pathway in LMIC (Figure 1) (80)}

Patients presenting with sepsis and septic shock to austere health care settings require an individualized and guarded approach regarding fluids and vasoactive agents with frequent re-assessment. However, regarding infection workup and antimicrobial administration, a protocolized approach is still likely to be extremely useful. The pathway (Figure 1) attempts to provide instruction on pediatric sepsis treatment in RLS with emphasis on the initial 2 hours (resuscitation), 2-6 hours, and 6-24 hours (postresuscitation), taking into consideration the wide microbial spectrum, potential resource constraints, the presence of a fluid-losing state, possible underlying chronic co-morbidity.

This physiologically-driven pathway is modified from the WHO algorithm for management of shock in areas with limited resources, with elements based on the authors' own work demonstrating a fluid-sparing effect of early norepinephrine use in pediatric septic shock $(44,81,82)$. However, this treatment pathway is untested in large trials, and we urge caution in its use.

Key points emphasized through the pathway stress early recognition, appropriate antimicrobial use while simultaneously addressing source control and reversing physiologic aberrations by restoring circulation with carefully-monitored fluids and/or vasoactives and oxygen. Frequent monitoring of the response to treatment is emphasized. Judiciously timed blood transfusion should be considered in unresolved shock depending on the patient condition and local cut-offs for transfusion.

Regarding the initial fluid bolus, where patients with 




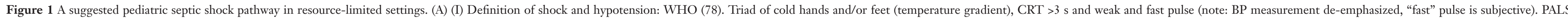

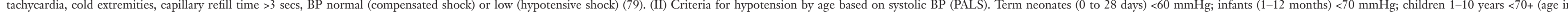

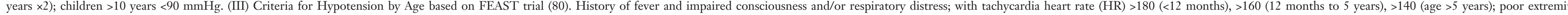

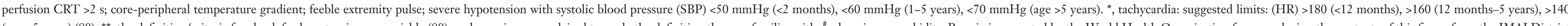

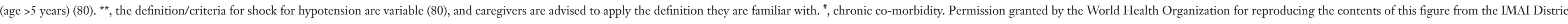

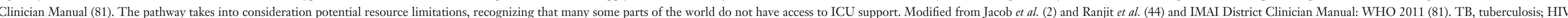

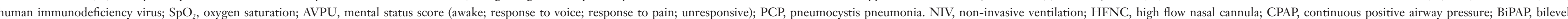
positive airway pressure. 


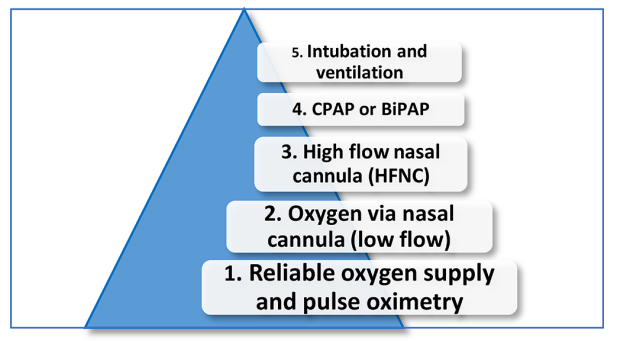

Figure 2 A suggested priority order for oxygen and respiratory support in LMIC. Modified from Inglis et al. (45).

clear history of fluid losses (diarrhoea, vomiting) or decreased intake must be prioritized for titrated modest volume fluid resuscitation. It is important to check and correct low levels of glucose depending on available facilities. If point-of-care monitoring of blood gases lactate and/or metabolic parameters such as calcium are present, these should be expeditiously performed.

In light of the risk of fluid-overload especially in patients with co-morbidities including anemia and malnutrition, a suggested option is to avoid the initial fluid bolus in the absence of a fluid-losing state, and, if the patient perfusion is impaired, initiate low dose vasoactive infusion depending on availability.

Regarding initial vasoactive-inotrope, studies conducted in India and Brazil comparing dopamine vs. epinephrine indicated that epinephrine may be associated with lower risk of mortality and more organ failure-free days among survivors, and norepinephrine or epinephrine are now considered as first choice of vasoactive support in pediatric sepsis (3). However, the initial choice depends on availability and familiarity, and in many parts of the world dopamine and epinephrine are the usual first-line agents since they are cheap and readily available (83).

A dilute concentration of the vasoactive medication may be infused via a secure peripheral or intraosseous line if central access is unavailable.

Clinical identification of fluid-overload (FO) is especially challenging in young children, in whom crackles may be absent even when gross pulmonary edema is present (3). Worsening respiratory mechanics, particularly increasing tachypnoea, chest X-Ray features of pulmonary edema, or increasing liver span may be the only evidence of progressive fluid overload. Even in low-income countries, some health-care facilities may have access and expertise for rapid bedside point-of-care ultrasound, which can be very useful to tailor fluid and vasoactive inotrope infusions.
Bedside echocardiography and ultrasound may be very helpful to assess early FO, as there is increasing evidence that a "full" inferior vena cava (IVC) with minimal respiratory variation indicates adequate intravascular volume (84). A "wet" lung profile may also confirm that further fluid bolus administration may be harmful.

In the event that a patient presents with septic shock without a history of fluid loss, alternatives to initial small fluid bolus include early initiation of an inotrope-vasoactive agent \pm small volume fluid bolus especially in regions without access to mechanical ventilation, where the ability to provide respiratory support in the event that the patient worsens may be not be available.

Abnormal electrolyte values, especially low glucose, and calcium levels as well as hypothermia and hypoxemia should be corrected during the initial resuscitation.

\section{Monitoring of cardiorespiratory response and titration of bemodynamic support}

Whether fluids or vasoactive infusions are the first approach to the treatment of septic shock, the caregiver must carefully assess the response by monitoring serial trends in the heart rate, systolic mean and diastolic arterial blood pressure, oxygen requirement and respiratory mechanics, as well as clinical markers of cardiac output, all of which are reasonably straightforward to monitor even in LMICs.

\section{Respiratory/ventilatory support for patients with septic shock in LMIC: pragmatic solutions (Figure 2)}

\section{Oxygen}

Supplemental oxygen is the most important support for patients with acute respiratory failure and pulse oximetry is mandatory to monitor this therapy (85). Nasal cannulae (NC) are useful options in many hospital wards in environments with limited personnel since lower nursing skills are required to use this device safely (45).

\section{Continuous positive airway pressure (CPAP)}

Three trials from LMICs have recently demonstrated that CPAP can improve survival in children $<5$ years, compared with oxygen delivered via standard nasal cannula, further CPAP can also minimize the requirement for invasive mechanical ventilation (86-88). 
High flow nasal cannula (HFNC) oxygen therapy is a very effective and promising respiratory support modality in LMIC among patients with moderately severe hypoxaemia, and can decrease the need for invasive mechanical ventilation since it can provide low-levels of CPAP as well as minimize dead-space, thereby eliminating carbon dioxide (89). Moreover, HFNC requires less training for safe use and is generally well tolerated without VAP risks.

The disadvantage of HFNC is the requirement for very high oxygen requirements which may be a heavy draw on a hospital's limited oxygen supplies, however, HFNC is an important target for exploration in LMIC (45).

Consideration should be given to a trial of high-flow nasal oxygen or mark noninvasive ventilation (NIV) for patients with moderately severe hypoxaemia before considering endotracheal intubation. While the WHO guidelines continue to support the use of HFNC oxygen, they emphasize close monitoring to recognize clinical deterioration that may require emergent intubation (90).

\section{Non-invasive ventilation (NIV)}

A 2018 systematic review and meta-analysis outlined a comprehensive review of noninvasive ventilation (NIV) in LMICs, including bubble CPAP and ventilator-delivered NIV in both children and adults (90).

NIV was reported to be a safe support in ICUs in Africa and South Asia. However, the greatest risk of NIV is the potential to for intubation to be delayed, leading to higher mortality, further, NIV requires significant training and close patient monitoring to deliver effectively. However NIV is feasible and safe in many LMICs, and warrants further investigation to use safely in these areas (90).

Intubation and controlled mechanical ventilation must be considered in those with decreased conscious level, or decreased airway protection, or those with significant cardio-respiratory decompensation.

Sedative and analgesic drugs along with positive pressure ventilation (PPV) may cause significantly found reduced preload resulting in worse hemodynamic instability in the peri-intubation period. Isotonic fluids and dilute solutions of vasoactive infusions should be readily available. Ketamine should be the preferred induction agent since it can maintain relative cardiovascular stability (3). However, low titrated doses should be used, since patients who are "catecholaminedepleted" are at risk for cardiovascular collapse. Induction with agents such as benzodiazepines and propofol can result in profound hypotension and should be avoided (16).
If intubation and ventilation are unavoidable, iatrogenic harm must be avoided by minimizing secondary infections, and efforts to expedite weaning and liberate the patient from the ventilator at the earliest opportunity. Moreover, discussions with the family must emphasize expectations that are realistic with regards to duration of ventilation, prognosis and costs and, in order to minimize risks of continuing support to the point of futility at an ongoing escalating cost that is usually borne by the family (45). This strategy has important implications in contexts where the provision of PPV even for a single day can result in catastrophic expenditure (91).

\section{Training and quality improvement programmes}

Championing low-cost high-impact quality improvement (QI) programmes

It is essential that the critical care leadership in LMIC relentlessly champion the meticulous implementation of simple QI measures that have large impact e.g., those that can greatly improve outcomes at minimal resources and cost. Two examples in an Indian pediatric cardiac-surgical ICU include establishment of a strong infection prevention programme in the ICU and implementation of a surgical safety checklist $(92,93)$. While some obstacles may have to be initially overcome to engage all concerned stakeholders successfully, once improvements become evident, implementation becomes easier.

\section{Education, training and mentorship of health professionals at many levels}

This is a invaluable investment because it empowers personnel, especially nurses. Nurse empowerment leads to improved outcomes through higher-quality care, ongoing education, improved infection control and a far greater motivation $(68,94)$.

\section{International Collaboration-developing partnerships}

Despite great differences in environments, a lot may be learnt from the established health care facilities in HIC.

The LMIC- HIC collaboration can successfully improve many aspects of patient care, and provide career growth opportunities for local researchers (95). However, care should be taken that such collaborative efforts are well-grounded with realistic goals within the LMIC hospital systems. 
The future is closer than you thought! Harnessing progress in innovative digital health technology in LMIC

Although the challenges to scaling up to high-quality intensive care services are numerous, the opportunities to creatively improvise in this field are even more and hold promise to move LMIC closer to HIC equity in global healthcare (96). Emerging digital health and innovative technology may offer a large part of the solution paving the way for the development of an inexpensive, scalable, and sustainable model of critical care in LMICs.

At the global level, collaborative efforts towards a lesssiloed approach to scaling and integrating digital health may provide the necessary leadership to enable innovative solutions to reach healthcare practitioners and patients in LMICs (97).

\section{Wide-spread penetration of mobile technology}

Ubiquitous tools and technologies such as smart phones, cloud computing, three-dimensional printers, crowdfunding and social media have given rise to frugal grassroots innovation and entrepreneurship (98). For example, India used its Information-technology (IT)-ability to leapfrog poor medical infrastructure. "India leapfrogged twice earlier, and went from no phone to smartphone, and from no books to the internet. Technology can make quality healthcare accessible at affordable prices to all our citizens even in the remotest corners of countries." (99).

The problem of limited clinical availability can be overcome using smart technology especially when identifying of the child at risk. A novel digital triage tool is being developed and clinically evaluated in Africa that aims to identify high-risk patients and reduce treatment times. This mobile health platform, will provide an individualized risk prediction in real-time to facilitate the rapid triage patients and institution of life-saving therapies for children in LMIC, where the concerned specialists may not always be available and sepsis deaths are common (100).

\section{Telemedicine}

Telemedicine as applied to critical care also referred to as tele-critical care (TCC) accommodates the concept that TCC services can be provided to locations beyond the physical confines of an ICU, or a hospital (101).

Digital health in the Philippines pioneered open-source telemedicine and mobile Health projects that connected remote patients with specialists in the Philippine General Hospital (PGH). This community-based approach delivering eHealth solutions has gathered momentum in recent years, as the lack of doctors, nurses and other allied health workers in underserved areas became more severe (102).

\section{Wearable technology}

Inexpensive wearable devices offer an affordable approach to monitoring of vital signs in LMICs, and when combined with artificial intelligence (AI), may potentially also improve patient care, not only within the hospital but also to provide monitoring after discharge of high-risk patients with sepsis and ARDS (103).

Wireless wearable smart patches continuously and automatically track a patient's vital signs and is able to flag any changes in patient's condition and alert caregivers to early-onset sepsis far sooner than may be done by traditional monitoring. Further, these "smart patches" also performs real-time monitoring of the patient's response to treatment. Developments in this technology may be useful for clinicians and nurses in LMIC, since it have the potential for providing earlier alerts of patient clinical deterioration than with manual monitoring alone, especially for clinicians and nurses who are battling overwhelming workloads (104).

\section{Artificial intelligence (AI) and machine learning models may improve early detection and complex decision making in sepsis}

In order to reduce the great clinical variability in sepsis treatment with less than optimal decisions often leading to poorer outcomes, a reinforcement learning agent, the Artificial Intelligence (AI) clinician was developed, which extracted knowledge from many patients' data that exceeds by many-fold the life-time experience of human caregivers and was able to learn optimal therapeutic approach by analyzing multiple treatment decisions. The authors reported that the "AI clinician model" was able to provide individualized treatment decisions for sepsis that could improve survival from sepsis (105).

\section{Frugal innovation: doing more with less for more}

Frugal innovation is the idea that more can be done for less for many more people globally. i.e., to create significantly 
more value while minimizing the use of resources (102).

The healthcare sector stands to benefit most from the concept of 'frugal medical technologies that are poised to disrupt the healthcare economy and will hopefully meet the unmet clinical needs of the world' $(106,107)$.

A brief outline of types of frugal innovation in medicine with examples include the following.

Lean tools refer to techniques that simplify and adapt existing technologies and provide healthcare innovations to many more at greatly reduced costs. For example, a low-cost toaster-sized ventilator (AGVA) costing 2,000 USD was developed in India by a neurosurgeon and robotic scientist and has been shown to provide safe ventilator support even in non-ICU environments. It is small $(\sim 3.5 \mathrm{~kg})$, portable, robust, compatible with temperature fluctuations, unstable power sources and can function even with oxygen from a cylinder and has a built-in air-compressor. The device is highly functional, hardy, and user-friendly (by intensivists, nurses, and even family members during home-use if required), and is especially relevant during the current COVID-19 pandemic. While not optimal for infants and smaller children, it's consumables are re-usable.

General Electric's MACi ECG (108) and Rice University's bubble CPAP (88) are prime examples of equipment stripped of superfluous functions enabling the costs to be reduced to $1 / 2-1 / 15^{\text {th }}$ that of the original counterparts. Beyond being simply low-cost these devices have many advantages: they are portable, easy to maintain, able to function in austere environments with inexpensive and accessible spare parts. Lean tools developed for LMIC are sometimes so cost-effective and efficient that they are even better than the original solutions used in HIC. For example, Chinese engineers at Siemens company have removed infrequently used settings and developed an inexpensive CT scanner. The resulting machine has cut the cost by a third and the technology has "reversely" spread in the United States (109).

Opportunistic solutions refer to the clever use of inexpensive widely available technologies to overcome "old problems". Evolving mobile phone technology and the internet can have radical possibilities, from improved adherence to antiretroviral therapy via a mobilephone text messages (110) to identifying drugs that are counterfeit (111). 3D printers may improve accessibility to medical devices ranging from low-cost prosthetics (112) to equipment spare-parts.

Contextualized adaptations refer to the re-purposing existing materials tools or techniques, for example, urine reagent strips used to evaluate cerebrospinal fluid (113) or synovial fluid (114) were found to be reasonably good diagnostic tests, that are cheap and usable in poorlyresourced environments.

\section{Ready for prime-time: rapid identification of infectious diseases, sepsis identification and antibiotic susceptibility testing}

While rapid sepsis diagnosis is ideal for targeted therapy and infection control, conventional laboratory approaches are expensive, labour-intensive, and associated with unacceptable time-delays. Recent advances in micronanotechnologies have facilitated the development of lowcost micro-fluidic point-of-care (POC) devices for the rapid diagnosis of malaria, sepsis, and HIV infections, and the authors discuss the challenges to practical implementation and future perspectives (115).

Antimicrobial resistance (AMR) is at the forefront of global importance and concern, with increasing mortality rates and rising economic burden. Inadequate antimicrobial stewardship has resulted in an increasingly diverse scale and range of AMR mechanisms, and tackling this effectively requires ongoing education and improved diagnostics. A study that employed inexpensive, commercially available screen-printed electrodes that were modified with an agarose-based hydrogel deposit that show rapid growth, with potential time to antibiotic susceptibility results that were $<45$ minutes, demonstrating a significant improvement on the current gold standard of 24-48 hours, potentially allowing doctors to be able to prescribe the correct antibiotic to a patient for an infection more quickly (116).

\section{Pediatric critical care and pediatric sepsis research gaps in LMIC}

In the last 2 decades intensive care medicine research has picked up the pace and countless randomised controlled trials have been performed, supplying clinical care with the much needed scientific foundation, discarding obsolete treatments, and saving lives (117). Unfortunately only $10 \%$ of global health-care research expenditure addresses conditions that affect the poorest $90 \%$ of the world's population, often referred to as the Research "10-90 gap" (118). Worldwide conformity in sepsis care is an elusive pipe dream; thus, we should accept that differences exist but foster research to improve systems and tailor best practices to local context. 


\section{Lessons from the pandemic}

In LMICs, the COVID-19 pandemic has drawn stark attention to the scarcity of vitally-important critical care resources; it has also emphasized the ongoing need for training of health-care workers, the need for establishing management approaches that match local resources, and research that is focused on the unique clinical practice in LMICs.

\section{Conclusions}

In LMIC settings, the burden of childhood mortality from preventable deaths remains unacceptably high and a large undocumented burden of sepsis and critical illness continues to exist.

The great heterogeneity of common critical care syndromes such as sepsis and ARDS has slowed down progress in identifying treatment targets and consequently the needs of critically ill patients in LMIC are often unmet, with some patients exposed to interventions that can even harm. Given the wide variation in resources, it may be unrealistic to expect similar targets and global consensus on all aspects of management, and local critical care leadership in various regions must tailor a pragmatic diagnostic and therapeutic approaches to a range of conditions in their settings (117). This approach will engage many more clinicians and benefit children afflicted with sepsis in both resource poor and rich environments.

The Millennium Developmental Goals era has witnessed great progress in child health which has been due to collaborations among the public, governments, as well as the private sectors. Avoiding preventable deaths will not only greatly reduce the disease burden and mortality but will also help improve life expectancy and thereby decrease birth rates, increase household productivity, and even have a favourable impact on the gross domestic product. Investments in critical care should be appropriate and effective rather than costor technology- intensive. Such investments, can have large dividends across many clinical specialties as well as have a favourable impact on the health outcomes of populations (119).

\section{Acknowledgments}

Funding: None.

\section{Footnote}

Provenance and Peer Review: This article was commissioned by the Guest Editors (Jan Hau Lee, Vijay Srinivasan, and Debbie Long) for the series "Pediatric Critical Care" published in Translational Pediatrics. The article has undergone external peer review.

Peer Review File: Available at http://dx.doi.org/10.21037/tp20-310

Conflicts of Interest: Both authors have completed the ICMJE uniform disclosure form (available at http://dx.doi. org/10.21037/tp-20-310). The series "Pediatric Critical Care" was commissioned by the editorial office without any funding or sponsorship. The authors have no other conflicts of interest to declare.

Ethical Statement: The authors are accountable for all aspects of the work in ensuring that questions related to the accuracy or integrity of any part of the work are appropriately investigated and resolved.

Open Access Statement: This is an Open Access article distributed in accordance with the Creative Commons Attribution-NonCommercial-NoDerivs 4.0 International License (CC BY-NC-ND 4.0), which permits the noncommercial replication and distribution of the article with the strict proviso that no changes or edits are made and the original work is properly cited (including links to both the formal publication through the relevant DOI and the license). See: https://creativecommons.org/licenses/by-nc-nd/4.0/.

\section{References}

1. Fleischmann C, Scherag A, Adhikari NKJ, et al. Assessment of global incidence and mortality of hospitaltreated sepsis current estimates and limitations. Am J Respir Crit Care Med 2016;193:259-72.

2. Jacob ST, Lim M, Banura P, et al. Integrating sepsis management recommendations into clinical care guidelines for district hospitals in resource-limited settings: The necessity to augment new guidelines with future research. BMC Med 2013;11:107.

3. Weiss SL, Peters MJ, Alhazzani W, et al. Surviving Sepsis Campaign International Guidelines for the Management of Septic Shock and Sepsis-Associated Organ Dysfunction in Children. Pediatr Crit Care Med 2020;21:e52-106.

4. Levy MM, Evans LE, Rhodes A. The Surviving Sepsis Campaign Bundle: 2018 update. Intensive Care Med 2018;44:925-8. 
5. Kissoon N, Carapetis J. Pediatric sepsis in the developing world. J Infect 2015;71:S21-6.

6. Kissoon N, Uyeki TM. Sepsis and the global burden of disease in Children. JAMA Pediatr 2016;170:107-8.

7. Dugani S, Veillard J, Kissoon N. Reducing the global burden of sepsis. Cmaj 2017;189:E2-3.

8. Rudd KE, Kissoon N, Limmathurotsakul Di, et al. The global burden of sepsis: Barriers and potential solutions. Crit Care 2018;22:232.

9. Available online: https://apps.who.int/gb/e/e_wha70.html

10. Peltola L, Goddia C, Namboya F, et al. Sepsis Knowledge of non-physician personnel in Africa: A crosssectional study in Malawian district hospitals. Med Klin Intensivmed Notfmed 2015;110:49-54.

11. Murthy S, Leligdowicz A, Adhikari N. Intensive Care Unit Capacity in Low-Income Countries: A Systematic Review. PLoS One 2015;10:e0116949.

12. Becker JU, Theodosis C, Jacob ST, et al. Surviving sepsis in low-income and middle-income countries: new directions for care and research. Lancet Infect Dis 2009;9:577-582.

13. Baker T, Rylance J. country. 27-31

14. Baker T. Critical care where there is no ICU: Basic management of critically ill patients in a low-income country. Updat Anaesthesia 2012;28:27-31.

15. Machado FR, Nsutebu E, AbDulaziz S, et al. Sepsis 3 from the perspective of clinicians and quality improvement initiatives. J Crit Care 2017;40:315-7.

16. Dondorp AM, Dünser MW, Schultz MJ. Sepsis management in resource-limited settings. 2019. Available online: https://www.springer.com/gp/ book/9783030031428

17. Schultz MJ, Dunser MW, Dondorp AM, et al. Current challenges in the management of sepsis in ICUs in resource-poor settings and suggestions for the future. Intensive Care Med 2017;43:612-24.

18. Vukoja M, Riviello ED, Schultz MJ: Critical care outcomes in resource-limited settings. Curr Opin Crit Care 2018;24:421-7.

19. Mandelzweig K, Leligdowicz A, Murthy S, et al. Noninvasive ventilation in children and adults in low- and lowmiddle income countries: A systematic review and metaanalysis. J Crit Care 2018;47:310-9.

20. Varghese GM, Raj D, Francis MR, Sarkar R, Trowbridge P MJ: Epidemiology \& risk factors of scrub typhus in south India. Indian J Med Res 2016;144:76-81.

21. Gopalakrishnan R, Sureshkumar D, Thirunarayan MA. Melioidosis: An emerging infection in India. J Assoc
Physicians India 2013;61:612-4.

22. White NJ, Pukrittayakamee S, Hien TT, et al. Malaria. Lancet 2014;383:723-35.

23. White NJ, Turner GD, Day NP DA. Lethal malaria: Marchiafava and Bignami were right. J Infect Dis 2013;208:192-8.

24. Ranjit S, Kissoon N, Shamaraoc S. Dengue viral infections and shock syndromes: An overview. J Pediatr Infect Dis 2009;4:107-17.

25. Livermore DM. Fourteen years in resistance. Int J Antimicrob Agents 2012;39:283-94.

26. Molton JS, Tambyah PA, Ang BSP, et al. The global spread of healthcare-associated multidrug-resistant bacteria: a perspective from Asia. Clin Infect Dis 2013;56:1310-8.

27. Laxminarayan R, Matsoso P, Pant S, et al. Access to effective antimicrobials: a worldwide challenges. Lancet 2016;387:168-75.

28. Fahad A, Alghannam A, Aslanpour Z, et al. A systematic review of counterfeit and substandard medicines in field quality surveys. Integr Pharm Res Pr 2014;3:71-88.

29. Alhazzani W, Møller MH, Arabi YM, et al. Surviving Sepsis Campaign: Guidelines on the Management of Critically Ill Adults with Coronavirus Disease 2019 (COVID-19). Berlin Heidelberg: Springer, 2020.

30. Luregn J. Schlapbach NK. Defining Pediatric Sepsis. JAMA 2018;172:313-4.

31. Weiss SL, Fitzgerald JC, Maffei FA, et al. Discordant identification of pediatric severe sepsis by research and clinical definitions in the SPROUT international point prevalence study. Crit Care 2015;19:325.

32. Haniffa R, Pubudu De Silva A, Weerathunga P, et al. Applicability of the APACHE II model to a lower middle income country. J Crit Care 2017;42:178-83.

33. Breuer RK HA: Identifying Hesitation and Discomfort with Diagnosing Sepsis: Survey of a Pediatric Tertiary Care Center. Pediatr Qual Saf 2018;3:e099.

34. Riviello ED. Improving outcomes for severe sepsis in Africa: One step closer. Crit Care Med 2014;42:2439-40.

35. Andrews B, Semler MW, Muchemwa L, et al. Effect of an early resuscitation protocol on in-hospital mortality among adults with sepsis and hypotension: A randomized clinical trial. JAMA 2017;318:1233-40.

36. Andrews B, Muchemwa L, Kelly P, et al. Simplified severe sepsis protocol: A randomized controlled trial of modified early goal-directed therapy in Zambia. Crit Care Med 2014;42:2315-24.

37. Maitland K, George EC, Evans JA, et al. Exploring mechanisms of excess mortality with early fluid 
resuscitation: Insights from the FEAST trial. BMC Med 2013;11:68.

38. Hippensteel JA, Uchimido R, Tyler PD, et al. Intravenous fluid resuscitation is associated with septic endothelial glycocalyx degradation. Crit Care 2019;23:259.

39. Byrne L, Obonyo NG, Diab SD, et al. Unintended consequences: Fluid resuscitation worsens shock in an ovine model of endotoxemia. Am J Respir Crit Care Med 2018;198:1043-54.

40. Monge García MI, Guijo González P, Gracia Romero M, et al. Effects of fluid administration on arterial load in septic shock patients. Intensive Care Med 2015;41:1247-55.

41. Long E, Babl FE, Oakley E, et al. Cardiac Index Changes with Fluid Bolus Therapy in Children with Sepsis - An Observational Study. Pediatr Crit Care Med 2018;19:513-8.

42. Wills BA, Nguyen MD, Ha TL, et al. Comparison of three fluid solutions for resuscitation in dengue shock syndrome. N Engl J Med 2005;353:877-89.

43. Perner A, Cecconi M, Cronhjort M, et al. Expert statement for the management of hypovolemia in sepsis. Intensive Care Med 2018;44:791-8.

44. Ranjit S, Natraj R, Kandath SK, et al. Early norepinephrine decreases fluid and ventilatory requirements in pediatric vasodilatory septic shock. Indian J Crit Care Med 2016;20:561-9.

45. Inglis R, Ayebale E, Schultz MJ. Optimizing respiratory management in resource-limited settings. Curr Opin Crit Care 2019;25:45-53.

46. Dugani S, Laxminarayan R, Kissoon N. The quadruple burden of sepsis. CMAJ 2017;189:E1128-9.

47. Dugani S, Kissoon N. Global advocacy needed for sepsis in children. J Infect 2017;74:S61-5.

48. Global Neonatal and Under-5 Mortality: WHO. Available online: https://www.who.int/gho/child_health/mortality/ mortality_under_five_text/en/

49. Kumar P, Singhal N. Mapping neonatal and under-5 mortality in India. Lancet 2020;395:1591-3.

50. Gavidia R, Fuentes S, Vasquez R, et al. Low Socioeconomic Status Is Associated with Prolonged Times to Assessment and Treatment, Sepsis and Infectious Death in Pediatric Fever in El Salvador. PLoS One 2012;7:e43639.

51. Jackson S, Mathews K, Pulanic D, et al. Risk factors for severe acute lower respiratory infections in children: a systematic review and meta-analysis. Croat Med J 2013;54:110-21.

52. Molyneux E, Ahmad S, Robertson A. Improved triage and emergency care for children reduces inpatient mortality in a resource-constrained setting. Bull World Health Organ 2006;84:314-9.

53. von Saint André-von Arnim AO, Attebery J, Kortz TB, et al. Challenges and priorities for pediatric critical care clinician-researchers in low- and middle-income countries. Front Pediatr 2017;5:277.

54. Molyneux E, Ahmad S, Robertson A. Improved triage and emergency care for children reduces inpatient mortality in a resource-constrained setting. Bull World Health Organ 2006;84:314-9.

55. Geldsetzer P, Williams TC, Kirolos A, et al. The Recognition of and Care Seeking Behaviour for Childhood Illness in Developing Countries: A Systematic Review. PLoS One 2014;9:e93427.

56. Cameron A, Ewen M, Ross-Degnan D, et al. Medicine prices, availability, and affordability in 36 developing and middle-income countries: A secondary analysis. Lancet 2009;373:240-9.

57. Duke T, Wandi F, Jonathan M, et al. Improved oxygen systems for childhood pneumonia: a multihospital effectiveness study in Papua New Guinea. Lancet 2008;372:1328-33.

58. von Saint André-von Arnim AO, Attebery J, Kortz TB, et al. Challenges and Priorities for Pediatric Critical Care Clinician-Researchers in Low- and Middle-Income Countries. Trop Pediatr 2012;58:389-93.

59. Favin M, Steinglass R, Fields R, et al. Why children are not vaccinated: a review of the grey literature. Int Health 2012;4:229-38.

60. Gavi. Available online: https://www.gavi.org/sites/default/ files/programmes-impact/our-impact/apr/Gavi-ProgressReport-2019_1.pdf

61. WHO 2018. Assessment report of the Global Vaccine Action Plan. Available online: https://www.who.int/ immunization/global_vaccine_action_plan/SAGE_GVAP_ Assessment_Report_2018_EN.pdf?ua=1

62. Kalantari N, Borisch B, Lomazzi M. Vaccination-A Step Closer to Universal Health Coverage. J Public Health (Bangkok) 2020. Available online: https://doi.org/10.1007/ s10389-020-01322-y

63. Agrawal A, Gandhe M, Gandhe S, et al. Study of length of stay and average cost of treatment in Medicine Intensive Care Unit at tertiary care center. J Heal Res Rev 2017;4:24.

64. Organization WH: Pocket book of hospital care for children: guidelines for the management of common childhood illnesses. 2nd ed. World Health Organization, 2013.

65. Baker T. Critical care in low-income countries. Trop Med 
Int Health 2009;14:143-8.

66. Schell C, Wärnberg M, Hvarfner A, et al. The global need for essential emergency and critical care. Crit Care 2018;22:284.

67. Baker T, Schell C, Petersen D, et al. Essential care of critical illness must not be forgotten in the COVID-19 pandemic. Lancet 2020;395:1253-4.

68. Slusher TM, Kiragu AW, Day LT, et al. Pediatric critical care in resource-limited settings-overview and lessons learned. Front Pediatr 2018;6:49.

69. Chimwaza W, Chipeta E, Ngwira A, et al. What makes staff consider leaving the health service in Malawi? Hum Resour Health 2014;12:17.

70. Adhikari NKJ, Rubenfeld GD. qSOFA score for patients with sepsis in low- and middle-income countries. JAMA 2018;319:2175-7.

71. Schell CO, Castegren M, Lugazia E, et al. Severely deranged vital signs as triggers for acute treatment modifications on an intensive care unit in a low-income country Health Services Research. BMC Res Notes 2015;8:1-7.

72. George EC, Walker AS, Kiguli S, et al. Predicting mortality in sick African children: The FEAST Paediatric Emergency Triage (PET) Score. BMC Med 2015;13:174.

73. Molyneux E. Paediatric emergency care in developing countries. Lancet 2001;357:86-7.

74. Fung JST, Akech S, Kissoon N, et al. Determining predictors of sepsis at triage among children under 5 years of age in resource-limited settings: A modified Delphi process. PLoS One 2019;14:e0211274.

75. The Pediatric Acute Lung Injury Consensus Conference Group. Pediatric Acute Lung Injury Consensus Conference Group. Pediatr Crit Care Med 2015;16:428-39.

76. Lazzeri C, Peris A. The Kigali modification of the berlin definition: A new epidemiological tool for ARDS? J Thorac Dis 2016;8:E443-5.

77. Riviello ED, Kiviri W, Twagirumugabe T, et al. Hospital Incidence and Outcomes of the Acute Respiratory Distress Syndrome Using the Kigali Modification of the Berlin Definition. Am J Respir Crit Care Med 2016;193:52-9.

78. World Health Organization: Guideline. Updates on Paediatric Emergency Triage, Assessment and Treatment: Care of Critically-Ill Children. Guidel Updat Paediatr Emerg Triage, Assess Treat Care Crit Child 2016.

79. Duff JP, Topjian AA, Berg MD, et al. 2019 American Heart Association Focused Update on Pediatric Advanced Life Support: An Update to the American Heart Association Guidelines for Cardiopulmonary Resuscitation and Emergency Cardiovascular Care. Circulation 2019;140:e904-14.

80. Houston KA, George EC, Maitland K. Implications for paediatric shock management in resource-limited settings: A perspective from the FEAST trial. Crit Care 2018;22:119.

81. World Health Organization. IMAI district clinician manual: hospital care for adolescents and adults: guidelines for the management of illnesses with limited-resources. Available online: https://www.who.int/hiv/pub/imai/ imai2011/en/

82. Becker JU, Theodosis C, Jacob ST, et al. Surviving sepsis in low-income and middle-income countries: new directions for care and research. Lancet Infect Dis 2009;9:577-82.

83. Mekontso Dessap A. Frugal innovation for critical care. Intensive Care Med 2019;45:252-4.

84. Arya B, Kerstein D, Leu CS, et al. Echocardiographic assessment of right atrial pressure in a pediatric and young adult population. Pediatr Cardiol 2016;37:558-67.

85. Graham HR, Bakare AA, Gray A, et al. Adoption of paediatric and neonatal pulse oximetry by 12 hospitals in Nigeria: a mixed-methods realist evaluation. BMJ Glob Health 2018;3:e000812.

86. Maitland K, Kiguli S, Opoka R, et al. Children's Oxygen Administration Strategies Trial (COAST): A randomised controlled trial of high flow versus oxygen versus control in African children with severe pneumonia. Available online: https://doi.org/10.12688/wellcomeopenres.12747.1

87. Chisti MJ, Salam MA, Smith JH, et al. Bubble continuous positive airway pressure for children with severe pneumonia and hypoxaemia in Bangladesh: An open, randomised controlled trial. Lancet 2015;386:1057-65.

88. Kawaza K, Machen H, Brown J, et al. Efficacy of a LowCost Bubble CPAP System in Treatment of Respiratory Distress in a Neonatal Ward in Malawi. PLoS One 2014;9:e86327.

89. Frat J-P, Thille A, Mercat A, et al. High-Flow Oxygen through Nasal Cannula in Acute Hypoxemic Respiratory Failure. N Engl J Med 2015;372:2185-96.

90. WHO. Updated guideline: paediatric emergency triage, assessment and treatment: care of critically-ill children. World Health Organization, 2016.

91. Ye Y, Zhu B, Jiang L, et al. A Contemporary Assessment of Acute Mechanical Ventilation in Beijing: Description, Costs, and Outcomes. Crit Care Med 2017;45:1160-7.

92. Singh S, Kumar R, Karimassery S, et al. Improving outcomes and reducing costs by modular training in 
infection control in a resource-limited setting. Int J Qual Health Care 2012;24:641-8.

93. Clark SC, Dunning J, Alfieri OR, et al. EACTS guidelines for the use of patient safety checklists. Eur J Cardiothorac Surg 2012;41:993-1004.

94. Kumar RK. Delivering pediatric cardiac care with limited resources. Ann Pediatr Cardiol 2014;7:163-6.

95. Molyneux E, Mathanga D, Witte D, et al. Practical issues in relation to clinical trials in children in low-income countries: Experience from the front line. Arch Dis Child 2012;97:848-51.

96. Turner H, Hao N, Yacoub S, et al. Achieving affordable critical care in low-income and middle- income countries. Br Med J Glob Heal 2019.

97. Labrique AB, Wadhwani C, Williams KA, et al. Best practices in scaling digital health in low and middle income countries. Global Health 2018;14:103.

98. Prabhu J. Frugal innovation: Doing more with less for more. Philos Trans R Soc A Math Phys Eng Sci 2017;375:20160372.

99. Available online: https://theconversation.com/ smartphones-in-india-how-to-get-1-25-billion-peopleonline-65137

100.Mawji A, Li E, Komugisha C, et al. Smart triage: triage and management of sepsis in children using the point-ofcare Pediatric Rapid Sepsis Trigger (PRST) tool. BMC Health Serv Res 2020;20:493.

101.Subramanian S, Pamplin JC, Hravnak M, et al. Tele-critical care: An update from the society of critical care medicine tele-ICU committee. Crit Care Med 2020;48:553-61.

102. Teleheath Philippines. Available online: https:// innovatemedtec.com/content/how-digital-health-isbridging-the-healthcare-gap-in-the-philippines

103. Kroll RR, McKenzie ED, Boyd JG, et al. Use of wearable devices for post-discharge monitoring of ICU patients: A feasibility study. J Intensive Care 2017;5:64.

104. Wearable patch early sepsis recognition. Available online: https://www.medicaldevice-network.com/news/earlydiagnosis-sepsis/

105.Komorowski M, Celi L, Badawi O, et al. The Artificial Intelligence Clinician learns optimal treatment strategies for sepsis in intensive care. Nat Med 2018;24:1716-20.

106. Bhargava B. Welcome to BMJ Innovations. BMJ Innov 2014;1:1-2.

107. Bhatti Y, Prime M, Harris M, et al. The search for the holy grail: Frugal innovation in healthcare from lowincome or middle-income countries for reverse innovation to developed countries. BMJ Innov 2017;3:bmjinnov-2016.

108. Emerging economies drive frugal innovation
Available online: https:/www.who.int/bulletin/ volumes/91/1/13-020113/en/

109. Innovation countdown 2030. Available online: https:// www.path.org/resources/innovation-countdown-2030reimagining-global-health/

110.Lester RT, Ritvo P, Mills EJ, et al. Effects of a mobile phone short message service on antiretroviral treatment adherence in Kenya (WelTel Kenya1): A randomised trial. Lancet 2010;376:1838-45.

111.Drug anti-counterfeiting technology reaches 1 million uses 2012. Available online: https://sproxil. com/2012/01/25/drug-anti-counterfeiting-technologyreaches-1-million-uses/

112.Zuniga J, Katsavelis D, Peck J, et al. Cyborg beast: A lowcost 3d-printed prosthetic hand for children with upperlimb differences. BMC Res Notes 2015;8:10.

113.Joshi D, Kundana K, Puranik A, et al. Diagnostic accuracy of urinary reagent strip to determine cerebrospinal fluid chemistry and cellularity. J Neurosci Rural Pract 2013;4:140-5

114. Ravaud P, Hudry C, Giraudeau B, et al. Rapid diagnosis of inflammatory synovial fluid with reagent strips. Rheumatology 2002;41:815-8.

115. Tay A, Khoo BL, Ebrahimi Warkiani M. Microfluidics for Fast and Frugal Diagnosis of Malaria, Sepsis, and HIV/ AIDS. In: Frugal Innovation in Bioengineering for the Detection of Infectious Diseases. Springer, 2018:57-75.

116. Hannah S, Addington E, Alcorn D, et al. Rapid antibiotic susceptibility testing using low-cost, commercially available screen-printed electrodes. Biosens Bioelectron 2019;145:111696.

117. The Lancet Respiratory Medicine. The future of critical care: lessons from the COVID-19 crisis. Lancet Respir Med 2020;8:527.

118. Global Forum for Health Research \& World Health Organization. (2004). The 10/90 (ten ninety) report on health research 2003-2004. Geneva: Global Forum for Health Research. Available online: https://apps.who.int/ iris/handle/10665/44386

119. Geiling J, Burkle FM Jr, West TE, et al. Resource-poor settings: response, recovery, and research: care of the critically ill and injured during pandemics and disasters: CHEST consensus statement. Chest 2014;146:e168S-77S.

Cite this article as: Ranjit S, Kissoon N. Challenges and Solutions in translating sepsis guidelines into practice in resource-limited settings. Transl Pediatr 2021;10(10):26462665. doi: $10.21037 /$ tp-20-310 